\title{
Comparative Analysis of Genetic and Chemical Differences between Four Berberis Herbs Based on Molecular Phylogenetic and HPLC Methods
}

\author{
Tu Feng, ${ }^{a}$ Huan Du, ${ }^{b}$ Hanting Chen, ${ }^{c}$ Qunying Xiao, ${ }^{a}$ Yang He, ${ }^{*, c}$ and Gang Fan ${ }^{*, b}$ \\ ${ }^{a}$ School of Ecological Engineering, Key Laboratory of Biological Resources and Ecological Remediation of Guizhou \\ Province, Collaborative Innovation Center of Wetland Eco-engineering of Guizhou Province, Guizhou University of \\ Engineering Science; Bijie 551700, China: ${ }^{b}$ School of Ethnic Medicine, Chengdu University of Traditional Chinese \\ Medicine; Chengdu 611137, China: and ' ${ }^{c}$ School of Medical Technology, Chengdu University of Traditional Chinese \\ Medicine; Chengdu 611137, China. \\ Received May 1, 2018; accepted September 8, 2018; advance publication released online September 27, 2018
}

In traditional Tibetan medicinal system, Berberis herbs mainly originate from the dried barks of Berberis kansuensis, Berberis dictyophylla, Berberis diaphana, and Berberis vernae. In this study, molecular phylogenetic method based on four markers (i.e., rbcL, internal transcribed spacer (ITS), ITS2, and psbA-trnH) and HPLC chemical analysis were used to evaluate the chemical and genetic differences between the four Berberis species. The results showed that the discriminatory power of ITS, ITS2 and psbA-trnH was low, but the $r b c L$ marker was highly effective and reliable for the species differentiation. The four Berberis species can be successfully classified based on phylogenetic analysis of the $r b c L$ sequences. Moreover, the results of chemical analysis showed that four main alkaloids (i.e., berberine, palmatine, magnoflorine, and jatrorrhizine) cannot be used as chemical markers for discrimination of the four Berberis species. These findings provide valuable information for distinguishing the four Berberis Tibetan herbs.

Key words Berberis herb; molecular phylogenetic method; alkaloid; species differentiation; interspecies variation

Berberis plants, belonging to Berberidaceae, are widely distributed in India, Pakistan, China, Japan, Europe, and South America. Some species of the genus Berberis have medicinal properties because of the presence of various active alkaloids. ${ }^{1)}$ In China, several Berberis species including B. kansuensis SchneID., B. dictyophylla FrAnch., B. diaphana Maxin., B. dasystachya Maxim., B. nullinervis YING, and B. vernae SchNeID. have been used for the treatment of diarrhea, urinary frequency, diabetes, trachoma, and nephritis for centuries in traditional Tibetan medicinal system. ${ }^{2)}$ Among them, $B$. kansuensis, $B$. dictyophylla, B. diaphana, and $B$. vernae are the most commonly used species. ${ }^{3)}$ At present, the four species are used as substitutes for each other in Tibetan traditional system of medicine because they have similar biological effects. However, interspecies variations may affect the quality stability and homogeneity of herbs. Therefore, it is necessary to investigate their genetic and chemical differences.

Molecular evolutionary analysis is a common method to reconstruct plant systematics and study genetic differences among species. ${ }^{4}$ At present, the molecular phylogeny of 79 taxa of Berberis reconstructed by the internal transcribed spacer (ITS) sequences has been reported, ${ }^{5)}$ but little attention was paid to the medicinal plants of genus Berberis. In the present study, phylogenetic analyses based on $r b c L$, ITS, ITS2, and psbA-trnH sequences were first used to study the relationships and differences among the four Berberis medicinal species.

At present, several alkaloids including berberine, magnoflorine and palmatine are considered to be the major bioactive compounds of Berberis herbs. ${ }^{1,6)}$ They have been proved to possess significant pharmacological activities such as antibacterial, anti-inflammatory and anti-diabetic effects, ${ }^{7,8}$ which are consistent with the biological activities of Berberis herbs. ${ }^{1,9)}$ Therefore, these alkaloids are usually used as markers to evaluate the herbal quality. ${ }^{6}$ ) During the past decades, several analytical methods, including HPLC and non-aqueous capillary electrophoresis, ${ }^{6,10)}$ have been applied for the quality evaluation of Tibetan Berberis herbs. In this study, in order to better control the quality of Berberis herbs and promote their rational application, molecular phylogenetic analysis and HPLC method were used to evaluate genetic and chemical differences between the four Berberis medicinal species.

\section{MATERIALS AND METHODS}

Plant Materials A total of 19 batches samples including 7 batches of $B$. kansuensis, 5 batches of B. dictyophylla, 4 batches of $B$. diaphana, and 3 batches of $B$. vernae were harvested from various growth areas in China. The cortex part was separated from the plant. The plant materials were identified by Professor Gang Fan, and their voucher specimens deposited in the herbarium of School of Ethnic Medicine, Chengdu University of Traditional Chinese Medicine, Chengdu, China. Detailed information of the samples is shown in Supplementary Material (Table S1).

HPLC Quantitative Analysis of Four Active Alkaloids HPLC analysis was carried out according to our previously reported method. ${ }^{6}$ Representative HPLC chromatogram is presented in Fig. S1. The concentrations of berberine, palmatine, magnoflorine, and jatrorrhizine in each sample were calculated using the established calibration curves made from the standard compounds.

DNA Extraction, PCR Amplification and Sequencing Total DNA was isolated directly from dried samples using a 
modified cetyl trimethylammonium bromide (CTAB) method. ${ }^{11)}$ The primers and PCR conditions were used according to our previously reported method. ${ }^{12)}$ The PCR amplification was performed in a GeneAmp PCR System 9700 with a volume of $25 \mu \mathrm{L}$. PCR products were ligated with the pEASY-Blunt cloning vector. The ligation products were transformed into Trans-T1 chemically competent cells and grown overnight on Luria Bertani or L-Broth solid medium (IPTG, X-gal, Amp). The positive colonies were identified by colony PCR and sequenced by Shanghai Invitrogen Biotechnology Co., Ltd. (China). The sequencing results were analyzed with Roche software version 2.5.3 and all products were sequenced by forward and reverse reactions for sequence confirmation. Then deposited in GenBank and accession numbers were from MH457107 to MH457125.

Data Analysis The sequences including outgroup and our aimed species were initially performed a complete alignment by Clustal X 1.81 ${ }^{13)}$ with default parameters. The alignment was validated again by eye to enhance the fitness. The best nucleotide substitution model of the final alignment was determined by jModeltest 2.1.4. ${ }^{14)}$

According to the best nucleotide substitution model, each phylogenetic tree was constructed from the final gene alignment with maximum likelihood (ML) and Bayesian inference (BI), respectively. Phylogenetic tree with ML method was implemented in RAxML 8.0.17 ${ }^{15)}$ with $G T R+G$ model for the final likelihood search and switching to the per-site rate category model during the fast bootstrapping with 1000 replicates. BI-based phylogenetic tree was conducted with MrBAYES 3.2.2 $2^{16)}$ and the nucleotide substitution model was chosen from the result of jModeltest, ${ }^{14)}$ and we run four concurrent Markov chain Monte Carlo (MCMC) calculations of five million generations while sampling once every 100 generations. The first $25 \%$ of sampled trees were regarded as unreliable samples and thus was discarded. The rest of $75 \%$ samples were used to generate a majority rule consensus tree. We repeated all MCMC runs twice to confirm the consistent approximation of the posterior parameter distributions.

Moreover, one-way ANOVA followed by Tukey's multiple comparison tests were done using GraphPad Prism software. Statistical significance was determined on a $95 \%$ probability level $(p<0.05)$. Partial least squares discriminant analysis (PLS-DA) and hierarchical cluster analysis (HCA) were applied to sort samples into groups. PLS-DA was performed using SIMCA-P software (version 11.5) with unit variance (UV) scaling, and HCA was done by using MetaboAnalyst 4.0 (http://www.metaboanalyst.ca/).

\section{RESULTS}

Chemical Analysis of the Main Alkaloids Using the developed HPLC quantitative method, four active alkaloids were determined simultaneously in the four Berberis herbs. Each sample was analyzed in triplicate to determine the mean content (Table S1 and Fig. 1). In order to study the differences of the four alkaloids among the four species, one-way ANOVA followed by Tukey's multiple comparison tests were performed. As shown in Fig. 1, B. kansuensis and B. dictyophylla contained more magnoflorine compared with $B$. diaphana. The jatrorrhizine content in $B$. vernae was obviously higher than that in B. dictyophylla and B. kansuensis. Compared with $B$. dictyophylla and $B$. diaphana, B. kansuensis showed obviously higher level of palmatine. Moreover, B. kansuensis contained significantly more berberine compared with $B$. dictyophylla.

In order to further determine the discriminating power of the four components, the quantitative data obtained by the HPLC method were also submitted to multivariate statistical
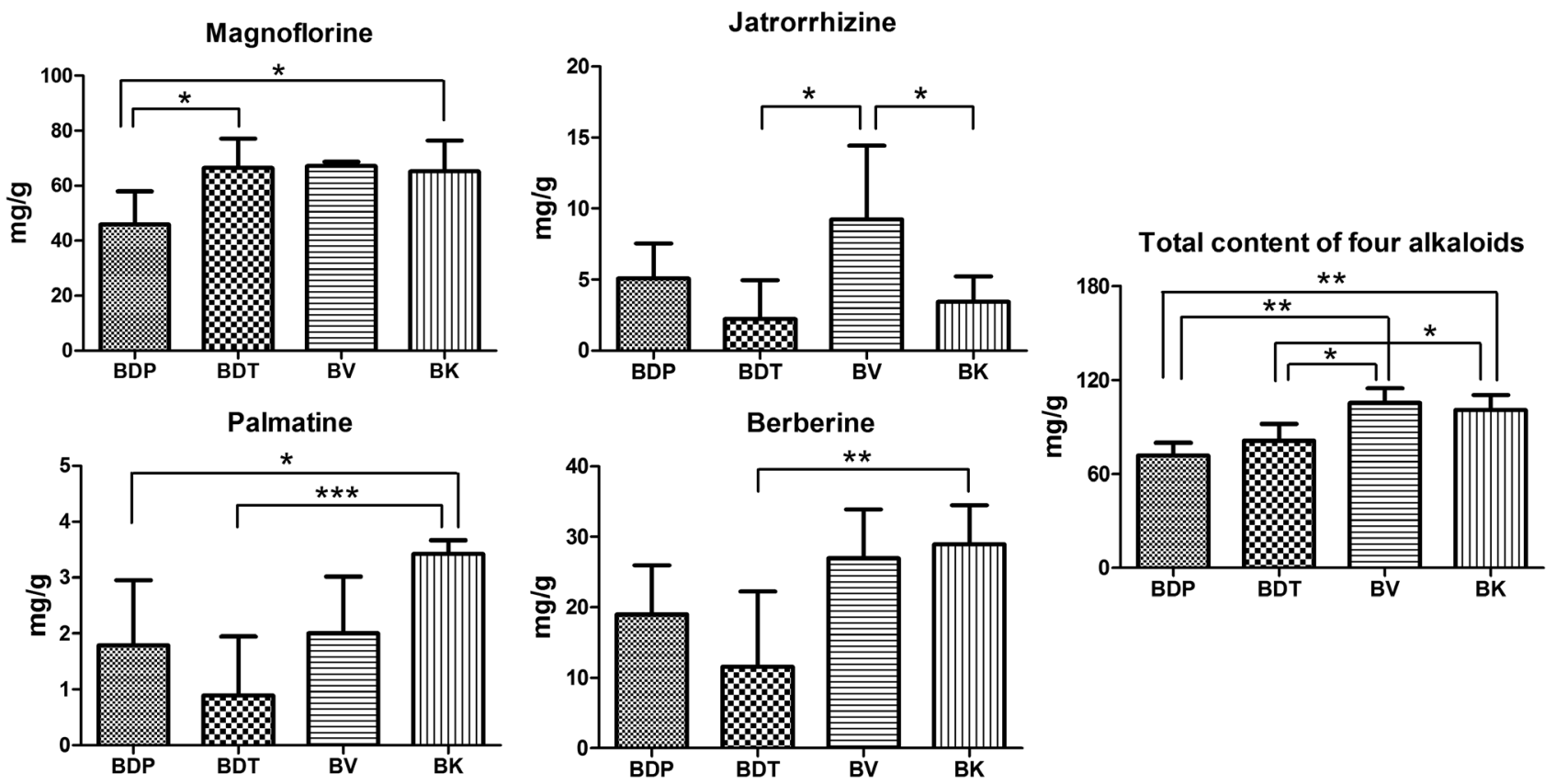

Fig. 1. Content (mg/g) of Four Alkaloids in B. diaphana (BDP), B. dictyophylla (BDT), B. vernae (BV), and B. kansuensis (BK) Determined by the HPLC Method

Data are given as mean \pm standard deviation (S.D.) ( $n=4$ for BDP, $n=5$ for BDT, $n=3$ for BV, and $n=7$ for BK). *,**, and *** indicate $p<0.05, p<0.01$, and $p<0.001$, respectively, based on Tukey's multiple comparison tests. 


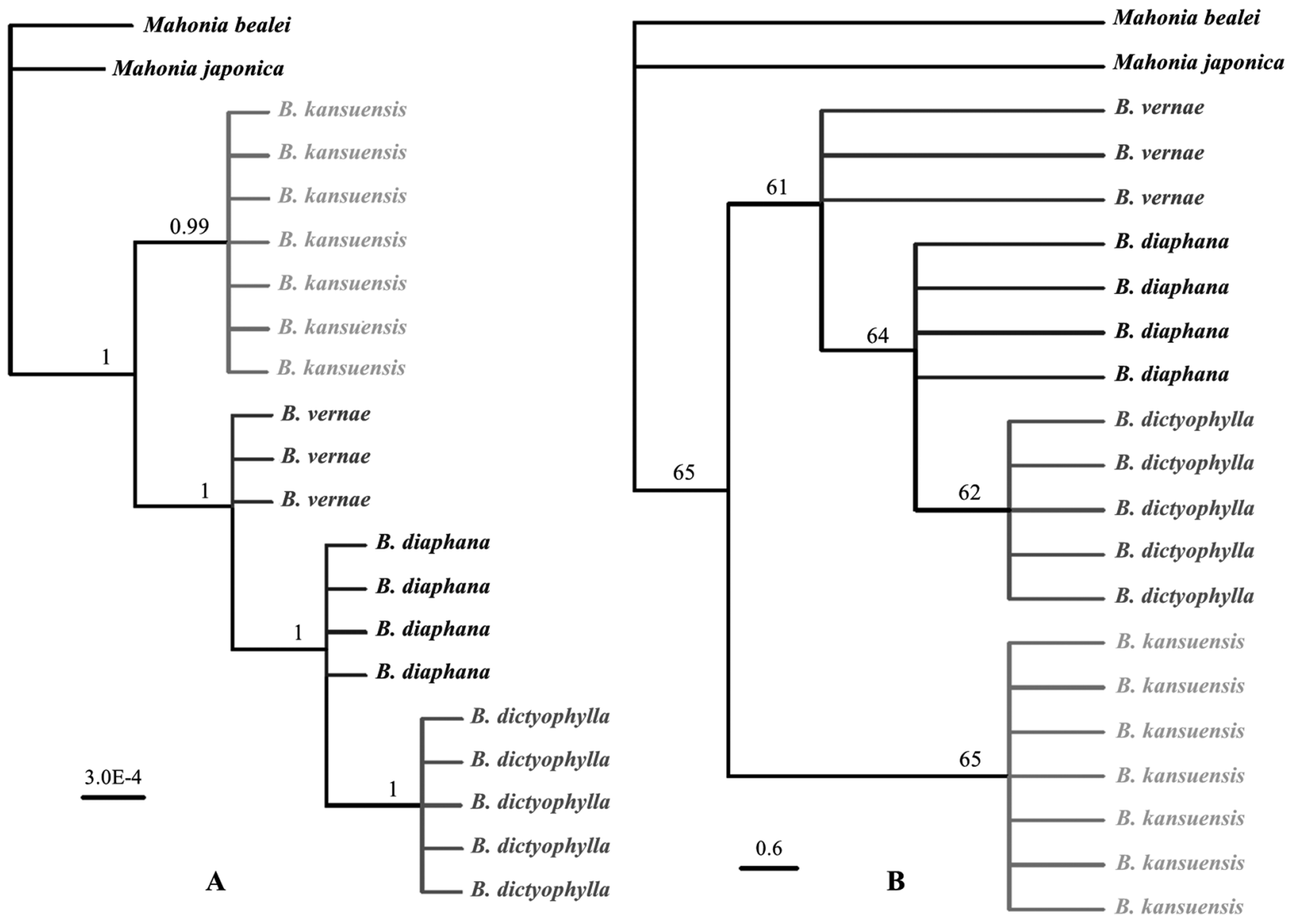

Fig. 2. Bayesian Inference (A) and Maximum Likelihood (B) Trees Derived from the $r b c L$ Marker

analysis. The PLS-DA score plot (Fig. S2) showed that the four species could not be differentiated by combining PLS1 with PLS2. Similarly, HCA analysis also obtained the same results, and samples of the four species were clustered together and could not be clearly distinguished (Fig. S3). These findings indicated that discrimination of the four Berberis herbs based on their phytochemicals is difficult.

Molecular Phylogeny Analysis Four candidate genes including $r b c L$, ITS, ITS2 and psbA-trnH from four species of $B$. dictyophylla, B. kansuensis, B. vernae and B. diaphana were selected to perform the rooted phylogenetic relationships construction. The outgroup species (Table S2) was chosen from the phylogenetic study of family Berberidaceae. ${ }^{17)}$ The species name and Genebank number was presented in Table S2. The best evolutionary model was used as presented in Table S3. According to the ourgroups and models, the phylogenetic relationships among $B$. dictyophylla, B. kansuensis, $B$. vernae, and $B$. diaphana were constructed by $\mathrm{ML}$ and $\mathrm{BI}$ (Fig. 2). The topology from both ML and BI was consistent that confirm the availability of our method used in this study. The most effective gene within the four species discrimination was $r b c L$, which basically cluster standard specimen from one species together. By contrast, the other genes could not distinguish our aimed species effectively and the species order in phylogenetic branches presented promiscuous (Figs. S4 and S5).

\section{DISCUSSION}

It is well known that the quality of herbal medicines is closely related to the levels of their active constituents. Alkaloids including berberine, magnoflorine, and palmatine are recognized as the main active constituents of Berberis herbs because their biological activities are consistent with the pharmacological effects of Berberis herbs. ${ }^{7-9)}$ In the present study, a reliable and accurate HPLC method was used for simultaneous quantification of berberine, palmatine, magnoflorine, and jatrorrhizine in the four Berberis species. The total content of the four alkaloids in B. kansuensis and $B$. vernae was obviously higher than that in $B$. dictyophylla and $B$. diaphana (Fig. 1). This indicates that the quality of B. kansuensis and $B$. vernae samples may be higher than the other two herbs. Considering these results, further studies are necessary to determine the quality differences between the four species by using pharmacodynamic method. Moreover, our studies demonstrated that the four alkaloids amounts differed dramatically $(p<0.05)$ between samples from different species. However, by combining PLS-DA and HCA results, we found that these four alkaloids cannot be used as chemical markers for discrimination of the four Berberis species.

On the other hand, 19 samples of Berberis herbs were collected to reconstruct the phylogeny for species evolution by $r b c L$, ITS, ITS2, and psbA-trnH sequences. The results showed that the four Berberis species can be successfully classified based on phylogenetic analysis of the $r b c L$ 
sequences. Although the ITS, ITS2 and psbA-trnH have been widely used as DNA markers for discrimination of medicinal plants ${ }^{18)}$ we found that the three markers were useless in distinguishing the four Berberis species. In addition, the ML and BI trees derived from the $r b c L$ marker (Fig. 2) indicated that $B$. dictyophylla, $B$. diaphana and $B$. vernae have closer phylogenetic relationship than to B. kansuensis. This may be due to geographic isolation and genetic evolution. However, the taxonomic relationship between these four species requires more research to explore.

In conclusion, a molecular phylogenetic method based on the $r b c L$ sequences was successfully developed for the differentiation of four Berberis medicinal plants. Although active ingredients (i.e., four alkaloids) do not have a key role in species discrimination, they are critical for the quality evaluation of Berberis herbs. The present study will provide valuable information for the species differentiation and quality control of four Berberis Tibetan herbs.

Acknowledgments The authors gratefully acknowledge the financial support from the National Natural Science Foundation of China (No. 81303310), the Science and Technology Foundation of Guizhou Province of China (No. [2010]2253), the Key Discipline of Ecology of Guizhou Province of China (No. ZDXK[2013]11), and the Science and Technology Special Fund of Sichuan Provincial Administration of Traditional Chinese Medicine (No. 2016Q060).

Conflict of Interest The authors declare no conflict of interest.

Supplementary Materials The online version of this article contains supplementary materials.

\section{REFERENCES}

1) Bhardwaj D, Kaushik N. Phytochemical and pharmacological studies in genus Berberis. Phytochem. Rev., 11, 523-542 (2012).

2) Health Bureau of Tibet, Qinghai, Sichuan, Gansu, Yunnan, and Xinjiang. Tibetan Medicine Standards. Qinghai People's Publishing Press, Xining, p. 11 (1979).

3) Zhang J, Lv XM, Li Y, Mo JQ, Wang MS, Lai XR, Fan G. Simultaneous determination of four alkaloids in different medicinal parts of
Berberidis Cortex by HPLC. Chin. J. Exp. Tradit. Med. Form., 23, 37-42 (2017).

4) Chen H, Fan G, He Y. Species evolution and quality evaluation of four Coptis herbal medicinal materials in Southwest China. 3 Biotech., 7, 62 (2017).

5) Kim YD, Kim SH, Landrum LR. Taxonomic and phytogeographic implications from ITS phylogeny in Berberis (Berberidaceae). $J$. Plant Res., 117, 175-182 (2004).

6) Li Y, Lv XM, Tang C, Lai XR, Zhang Y, Fan G. Quality evaluation of cortex berberidis from different geographical origins by simultaneous high performance liquid chromatography combined with statistical methods. Trop. J. Pharm. Res., 15, 1973-1981 (2016).

7) Tang LQ, Wei W, Chen LM, Liu S. Effects of berberine on diabetes induced by alloxan and a high-fat/high-cholesterol diet in rats. $J$. Ethnopharmacol., 108, 109-115 (2006).

8) Grycová L, Dostál J, Marek R. Quaternary protoberberine alkaloids. Phytochemistry, 68, 150-175 (2007).

9) Srivastava S, Srivastava M, Misra A, Pandey G, Rawat AKS. A review on biological and chemical diversity in Berberis (Berberidaceae). EXCLI J., 14, 247-267 (2015).

10) Gao WH, Lin SY, Jia L, Guo XK, Chen XG, Hu ZD. Analysis of protoberberine alkaloids in several herbal drugs and related medicinal preparations by nonaqueous capillary electrophoresis. J. Sep. Sci., 28, 92-97 (2005).

11) Doyle JJ, Doyle JL. A rapid DNA isolation procedure for small quantities of fresh leaf tissue. Phytochem. Bull., 19, 11-15 (1987).

12) He Y, Hou P, Fan G, Song Z, Arain S, Shu H, Tang C, Yue Q, Zhang Y. Authentication of Angelica anomala Avé-Lall cultivars through DNA barcodes. Mitochondr. DNA, 23, 100-105 (2012).

13) Thompson JD, Gibson TJ, Plewniak F, Jeanmougin F, Higgins DG. The CLUSTAL $\mathrm{X}$ windows interface: Flexible strategies for multiple sequence alignment aided by quality analysis tools. Nucleic Acids Res., 25, 4876-4882 (1997).

14) Posada D. jModelTest: Phylogenetic model averaging. Mol. Biol. Evol., 25, 1253-1256 (2008).

15) Stamatakis A. RAxML version 8: a tool for phylogenetic analysis and post-analysis of large phylogenies. Bioinformatics, 30, 13121313 (2014).

16) Ronquist F, Huelsenbeck JP. MrBayes 3: Bayesian phylogenetic inference under mixed models. Bioinformatics, 19, 1572-1574 (2003).

17) Wang W, Chen ZD, Liu Y, Li RQ, Li JH. Phylogenetic and biogeographic diversification of Berberidaceae in the northern hemisphere. Syst. Bot., 32, 731-742 (2007).

18) Techen N, Parveen I, Pan Z, Khan IA. DNA barcoding of medicinal plant material for identification. Curr. Opin. Biotechnol., 25, 103110 (2014). 